\title{
Desarrollo para los medios y acceso a la información en una sociedad post-conflicto: notas sobre Timor Oriental
}

DOI: $10.32870 /$ mycp.v14i41.368

Frida Rodelo Amezcua ${ }^{1}$

\section{Resumen}

$\mathrm{E}$

n Timor Oriental se han implementado diferentes tipos de estrategias e iniciativas para el desarrollo de los medios. El caso de Timor Oriental ejemplifica la relación entre medios y conflicto, aspecto que ha preocupado a actores internacionales y académicos por la capacidad de los medios tanto de empeorar el estado de estas situaciones como de desempeñar un papel positivo.

Palabras clave: desarrollo para los medios, legislación de los medios, Timor Oriental.

\section{Abstract}

East Timor has been the recipient of different kinds of strategies and initiatives on media development. This case exemplifies the relationship between media and conflict, a subject that has concerned international actors and scholars because of the media's potential either to worsen this kind of situations or have a positive role.

Keywords: media development, media policy, East Timor.

1. Maestra en Ciencias Sociales por la Universidad de Guadalajara. Correo electrónico: viri.rodelo@gmail.com. Los textos citados cuya lengua original es el inglés fueron traducidos por la autora. 


\section{Introducción}

En Timor Oriental, la nación más joven del mundo, los conflictos armados así como el subdesarrollo han impuesto retos importantes para los medios locales: éstos deben lograr no solamente ser democráticos y responsables sino también accesibles para la población y sostenibles. En un informe reciente, el Programa de Naciones Unidas para el Desarrollo (UNDP, 2007: 5) indicó que la falta de acceso a las noticias y la información en este país, sobre todo en las zonas del interior, ha llevado a "un sentimiento creciente de aislamiento y frustración así como estimulado la circulación de rumores". Este artículo pretende situar al lector en el contexto de este pequeño país del sureste asiático y presentar las soluciones que actores locales y extranjeros han propuesto e implementado para atender este problema.

El área de mejora para los medios en el marco de la ayuda para el desarrollo internacional supone que la combinación de malas prácticas por parte de los medios noticiosos (como propagar rumores, discursos de odio, sesgos y sensacionalismo) y la fragilidad del Estado pueden llevar a la desestabilización política, brotes de violencia y pánico social. Por lo tanto, los medios deben ser especialmente precisos, pertinentes y responsables durante situaciones delicadas, entre las cuales podemos enumerar crisis alimentarias y políticas, emergencias sanitarias, desastres naturales, procesos electorales y conflictos violentos.

Por otra parte, existe una vasta tradición de pensamiento liberal que enaltece la importancia de la libertad de prensa como fortalecedora de la democracia a través de diferentes mecanismos, como incentivar la rendición de cuentas. También diversos actores e instituciones consideran que un medio noticioso con periodistas bien entrenados puede generar efectos positivos. Por ejemplo, Hyer y Covello, en un manual para la Organización Mundial de la Salud, enlistan las siguientes maneras en las que los medios pueden ayudar durante una emergencia:

Informar y educar; sacar la noticia rápidamente; llegar a las audiencias más importantes; concentrar apoyos; funcionar como un watchdog público (por ejemplo, al cuestionar acciones o recomendaciones); ayudar a prevenir el miedo excesivo y la ansiedad; proporcionar la información precisa y necesaria; corregir la información errónea; estimular los comportamientos que son propicios; y calmar al público nervioso (Hyer y Covello, 2005: 1). 
Bratic y Schirch (2007: 9-11), a su vez, reconocen el rol de los medios durante conflictos en áreas como proporcionar e interpretar información, fungir como perro guardián (watchdog) y guardabarreras (gatekeeper), influenciar las políticas públicas, proporcionar un canal para las negociaciones entre los diferentes grupos, promover la paz y disminuir la polarización entre los grupos. Norris y Zinnbauer (2002: 13) resaltan la importancia de fortalecer los "canales de comunicación" para la rendición de cuentas y el diálogo democrático en las democracias electorales en proceso de formación o reformación. Estos autores encontraron correlaciones significativas entre indicadores de libertad de expresión, acceso a medios y comunicaciones e indicadores

En Timor Oriental, la nación más joven del mundo, los conflictos armados así como el subdesarrollo han impuesto retos importantes para los medios locales: éstos deben lograr no solamente ser democráticos y responsables sino también accesibles para la población y sostenibles de buen gobierno para 135 países del mundo. Los autores aclaran, además, que el periodismo independiente es importante pero sólo funciona si reúne dos condiciones: (1) independencia de los canales de comunicación de masas y (2) acceso público generalizado a estos medios (2002: 9).

\section{El desarrollo para los medios dentro del marco de la ayuda internacional para países con conflicto}

Como hemos visto, la categoría de "desarrollo para los medios" dentro del marco de la ayuda internacional para el desarrollo ha tenido entre sus fundamentos principales: (1) la hipótesis de que los medios pueden tener un efecto positivo indirecto en el nivel de desarrollo humano y (2) evitar que malas prácticas de los medios agraven un conflicto -e inclusive, en palabras de Howard (2009: 7-8), "influenciar la opinión y el comportamiento públicos hacia la resolución no violenta del conflicto".

Respecto al último punto, de acuerdo con Price (2000: 1-2), después de los eventos de Ruanda y Yugoslavia en los que fueron transmitidos mensajes de incitación al genocidio a través de medios masivos, organizaciones guberna- 
mentales internacionales (OGI) así como organizaciones no gubernamentales internacionales (ONGI) estuvieron interesadas en acciones de prevención, detención de este tipo de actividades y mejoramiento de la comunicación en escenarios de conflicto. Para Price (2000: 2-3), hubo dos maneras "probadas" de intervención de comunicación dirigidas a detener la propaganda de guerra durante y después de conflictos armados: crear medios alternativos directamente operados por las OGI "por lo menos inicialmente" y ayudar al desarrollo para los medios ya existentes.

Un ejemplo de medios directamente operados por las OGI son las estaciones de radio instaladas por las misiones de Naciones Unidas en las zonas de conflictos armados. Bratic (2005: 113-114) señala que la primera estación de este tipo fue Radio UNTAC, administrada por la misión UNTAC en Camboya, aunque habían habido intentos anteriores de hacer lo mismo en otros conflictos (véase Orme, 2010).

La segunda medida, ayudar al desarrollo para los medios nativos ya existentes, va dirigida hacia el fortalecimiento de la sociedad civil: para Price (2000: 2-3) es una opción menos "controladora", se enfoca en los medios que "introducen una voz nueva", y tiene "la esperanza de construir una esfera pública, una sociedad civil y una maquinaria de largo plazo para la paz y la reconstrucción", así como de formar "un patrimonio de información apartidista, el establecimiento de la infraestructura para el pluralismo y la emergencia de un electorado informado" (Price, 2000: 2-3).

El área de desarrollo para los medios ha sido incluida desde 1990 (Gillete, 2006: 1) en los programas de cooperación internacional de países donantes en las "democracias emergentes", primero en Europa central y del este y la antigua Unión Soviética. De acuerdo con Gillete (2006: 1), los primeros en implementar programas de desarrollo para los medios fueron la agencia de Estados Unidos para el desarrollo internacional (USAID, por sus siglas en inglés), el Departamento de Estado norteamericano y sus contrapartes europeas y canadiense. El discurso de los donantes de ayuda para el desarrollo de los medios, por lo menos en el caso de USAID, es que una democracia sana requiere del acceso a la información porque esto asegura que los ciudadanos tomen decisiones informadas y además da oportunidad para que éstos revisen que los representantes electos hayan cumplido con sus responsabilidades (USAID, 1999: 3).

Gillette (2006) encuentra dos aspectos diferentes pero igualmente importantes en el desarrollo para los medios después de un conflicto: los 
relacionados con los medios (es decir, con los periodistas, los estudiantes de periodismo, las empresas de medios, las asociaciones de periodistas y la ética en el periodismo) y los relacionados con el entorno de los medios, es decir, "construir un entorno legal e institucional para proteger, profesionalizar y, de forma cuidadosa y limitada, regular a los medios" (Gillette, 2006: 1). ${ }^{2}$

Siguiendo con la distinción de Gillette, Price y Krug (2002: 5) encuentran entre los componentes del entorno posibilitador (enabling environment) un balance entre medios públicos y privados, entorno de competencia entre los medios, acceso y derecho a impartir información, Estado de derecho, acceso a la información, libertad para ejercer el periodismo sin necesidad de una licencia, protección para los periodistas en ejercicio de su actividad profesional, una regulación de contenidos que sea democrática, acceso a los nuevos medios, una sociedad civil sana — una en la que ésta es activa-, educación sobre la importancia de los derechos, además de otros factores adicionales como, por ejemplo, la economía y la alfabetización.

Aunque el entorno legal es sumamente importante, los propios autores reconocen que no es lo único necesario para brindar la madurez, independencia y desarrollo que requieren los sectores de medios:

[...] discutimos leyes específicas que son importantes ladrillos de construcción. Aun así, enfatizamos los alrededores de la ley y la creación de una cultura de medios independientes y plurales. Después de todo, ¿qué es lo que hace que una sociedad sea abierta y tolerante y otra no? ¿Qué es lo que produce una ciudadanía que no solamente tiene las fuentes para estar informada sino que además las aprovecha? Es más fácil y claro ver cuáles son los pasos hacia atrás que imposibilitan que una cultura como esa se desarrolle en una sociedad. Las herramientas de represión del discurso son más fáciles de identificar que aquellas que fomentan la productividad y el uso de la información. Leyes de medios apropiadas por sí solas no hacen que una sociedad civil exista, aunque un marco legal puede ser beneficioso (Price y Krug, 2002: 5).

Como parte de las últimas operaciones internacionales para construcción de la paz (peacebuilding) en conflictos armados, las OGI han incluido el "fortale-

2. Por ejemplo, el propio Gillete fue el Comisionado Temporal para los Medios de Kosovo de la UNMIK. Esta institución tenía "el poder de imponer multas de hasta 50 mil euros por violación [del código de ética] y un mandato amplio aunque vago de producir medios independientes y profesionales en Kosovo e implementar un régimen regulatorio temporal para todos los medios en Kosovo" (Gillete, 2006). 
cimiento" o el "desarrollo" para los medios democráticos en sus agendas de actividades de gobernanza democrática.

$\mathrm{Al}$ igual que en el tema de la ayuda internacional para el desarrollo en general, el cumplimiento de metas de ayuda para el desarrollo de los medios requiere prever el aspecto de la sustentabilidad y los efectos adversos que produce la ayuda externa en la economía local y en la cultura. Muchos proyectos incluyen la donación de instalaciones y equipo que después del término del proyecto pueden descomponerse, ser robados, necesitar mantenimiento o simplemente ser económicamente insostenibles. Para Waisbord (2007), "la falta de sustentabilidad quiere decir que los resultados de corto plazo no se traducen en ganancias a largo plazo". Waisbord también sugiere que los actores locales deben liderar los programas y que las prioridades y acciones globales y locales deben estar alineadas.

\section{El conflicto de Timor Oriental}

Timor Oriental es al día de hoy una nación relativamente estable del sureste asiático que aún sufre de tensiones políticas después de haber sufrido un conflicto armado. El país ha recibido una cantidad importante de ayuda internacional para el desarrollo durante los últimos diez años.

La parte oriental de la isla de Timor era conocida simplemente como el "Timor Portugués", mientras que la otra parte de la isla fue en tiempos coloniales el “Timor Holandés". De ser una colonia portuguesa, fue invadida en 1975 por tropas indonesias. Ya como provincia indonesia, numerosas atrocidades fueron cometidas en contra de la población, lo que dio como resultado por lo menos 150 mil muertes, entre homicidios y víctimas de hambruna, durante el periodo de ocupación (1975-1999), según las propias cifras oficiales indonesias (Durand, 2009: 149).

El fin del mandato del dictador indonesio Suharto — quien fue obligado a renunciar en mayo de 1998-, las luchas del pueblo timorense (la guerrilla y acciones civiles) y negociaciones ante Naciones Unidas y entre Portugal e Indonesia fueron algunos factores que incidieron en que a este pueblo se le permitiera elegir su destino a través de una consulta popular (Durand, 2009: 139-144). La consulta popular fue organizada por la misión de Naciones Unidas en Timor Oriental (United Nations Mission in East Timor, UNAMET) y llevada a cabo en agosto de 1999. Esta consulta ofrecía dos opciones: ser una provincia autónoma de Indonesia o formar un Estado independiente. La 
mayor parte de los timorenses escogió la independencia. Sin embargo, hubo brotes de violencia comenzados por milicias financiadas y entrenadas por indonesios que estaban a favor de la autonomía (Durand, 2009: 144-146). Estas milicias empezaron un conflicto muy violento de grandes proporciones en el que fueron quemados infraestructura, edificios y casas y en el que más de cien mil personas fueron desplazadas. Después de algunos días, una fuerza multinacional fue autorizada para asistir en el conflicto. El mandato de la administración transicional de Naciones Unidas en Timor Oriental (United Nations Transitional Administration in East Timor, UNTAET) duró desde el 25 de octubre de 1999 hasta el 20 de mayo de 2002, cuando la independencia de la República Democrática de Timor Oriental fue restaurada. ${ }^{3}$ Las primeras elecciones presidenciales se llevaron a cabo en abril de 2002.

Después de un brote de violencia en 2006, Timor Oriental solicitó una vez más asistencia militar y policiaca internacional y las Naciones Unidas extendieron su presencia en el país. Un grupo de militares timorenses se habían quejado en enero de ese año, en una petición ante el presidente del país, de sus condiciones de trabajo. De acuerdo con Hicks (2009: 85), tras no ver cumplidas sus pretensiones, estos militares abandonaron sus puestos $y$ posteriormente fueron destituidos de sus cargos. Lo que siguió fue una serie de choques violentos entre la policía nacional, los militares levantados y grupos de jóvenes. La misión integrada de Naciones Unidas en Timor Oriental (United Nations Integrated Mission in Timor-Leste, UNMIT) se estableció en agosto de 2006. Irónicamente, mucha de la tensión desapareció en 2008, después de que tanto el presidente como el primer ministro de Timor Oriental fueron agredidos en intentos separados de homicidio y en los que el principal jefe de los peticionarios, Alfredo Reinado, murió. Contrariamente a lo que sucedió en 2006, estos incidentes no llevaron a la desestabilización del país, y, además, el área de gobernanza democrática de UNMIT pudo asistir al gobierno timorense en la exitosa organización de los procesos electorales de 2007 (elecciones presidenciales) y 2009 (elecciones locales).

De lo anterior tenemos que tras sufrir conflictos constantes, guerra sucia y ocupación por décadas, Timor Oriental es actualmente una joven nación de poco más de un millón de habitantes con un índice medio de desarrollo hu-

3. "Restauración" porque, ante la presión de la invasión indonesia, el Frente Timorense de Liberación Nacional (Fretilin) había proclamado la independencia unilateralmente el 28 de noviembre de 1975. 
mano (0.502, en el lugar 120 de entre 169 países; UNDP, 2010) ${ }^{4}$ y deficiencias en áreas como salud, educación, sanidad, seguridad alimentaria y caminos. Otros problemas que tienen relación estrecha con el conflicto armado son el desorden en la tenencia de la tierra, la persistencia del paramilitarismo y de grupos de choque y las diferencias entre la sociedad. Con "diferencias" me refiero a las que suelen existir en las sociedades post-conflicto entre los "ganadores" y los "vencedores", pero también a la distinción entre ema lorosa'e (los timorenses de la parte oriental del país) y ema loromonu (los de la parte occidental). En el conflicto de 2006, mencionado anteriormente, el grupo del ejército levantado estaba compuesto por militares provenientes de distritos del oeste que protestaban contra lo que consideraban actos de discriminación. Al extenderse este conflicto a peleas entre pandillas y otros actos de violencia, todo el país se involucró en esta distinción geográfica (Hicks, 2009).

Como Estado independiente, Timor Oriental ha sobrevivido económicamente gracias a las ganancias derivadas de la explotación de pozos de petróleo y gas natural por parte de compañías extranjeras. De acuerdo con La'o Hamutuk (2011, 3 de mayo: 4), 95\% de los gastos gubernamentales son financiados por las ganancias petroleras.

En cuanto a lo cultural, una situación particular que limita el acceso a la información es la referente a los niveles de analfabetismo y el multilingüismo. Solamente $50.1 \%$ de la población timorense con 15 años o más puede leer y escribir (UNDP, 2009a), lo que limita aún más el acceso a la información escrita y corona a la radio como el sector de medios con mayor penetración y crecimiento del país. El tetun, idioma oficial del país y hablado por más de 80\% de la población del país (Taylor-Leech, 2008), es la lingua franca de Timor Oriental pero es considerada insuficientemente desarrollada para ser soporte del sistema jurídico y la educación superior y, además, una buena parte de los timorenses no la saben escribir porque no aprendieron en la escuela a escribir tetun sino indonesio. El país tiene además una gran diversidad lingüística; se han identificado por lo menos otros 16 lenguajes nativos en el país (Taylor-Leech, 2008: 155). El portugués, el otro idioma oficial, se usa en el sistema jurídico y en la educación superior. Es hablado principalmente por una proporción de la población de los adultos mayores porque estaba prohibido durante la ocupación indonesia, aunque tuvo un papel político "vital"

4. En cuanto a su extensión territorial, Timor-Leste $\left(14874 \mathrm{~km}^{2}\right)$ es un poco más grande que Bahamas y más pequeño que el estado mexicano de Hidalgo. 
porque fue un lenguaje usado por el movimiento clandestino de resistencia (Taylor-Leech, 2008: 157). El uso del portugués añade un reto adicional para los medios timorenses pues muy pocos periodistas lo hablan (inclusive muchos legisladores no lo dominan, ETLJB, 2010, julio), además de complicar aún más el acceso a la justicia. Por otra parte, el indonesio, con categoría de "idioma de trabajo", es hablado y entendido por casi la mitad de población, principalmente los jóvenes. Aún tiene un papel importante en la educación y en el trabajo pues muchos jóvenes timorenses saben leer y escribir en este idioma (muchos de ellos estudiaron en Indonesia). El inglés, el otro "idioma de trabajo", es hablado por una proporción pequeña de la población. Desempeña un papel importante en el sector de servicios y la diplomacia principalmente debido a la proximidad y la importancia política de Australia, así como la presencia ubicua de expatriados de ese país.

\section{El sector de medios en Timor Oriental: empezar desde cero}

Antes de 1999, el único periódico editado en la provincia indonesia de Timor Timur era La Voz de Timor Oriental (Suara Timor Timur en indonesio). Reportes mencionan además a la estación católica Radio Timor Kmanek (Article 19 and Internews 2005: 44), fundada en 1994, y a la Radio Republik Indonesia. ${ }^{5}$ En este periodo, UNAMET organizó la previamente referida consulta popular de 1999 con diversas dificultades: se tenía muy poco tiempo para organizar la consulta, los timorenses eran intimidados por parte de las milicias pro-autónomas y la condición de los caminos era mala. UNAMET lanzó una campaña de información pública e instaló una estación de radio que inició transmisiones el 13 de junio para propagar información sobre la consulta popular: comunicados de prensa diarios y programas de educación electoral.

No quedó ningún medio en operación en Timor Oriental tras la violencia de 1999: las oficinas del Suara Timor Timur fueron saqueadas y destruidas y lo mismo sucedió con la infraestructura telefónica, eléctrica y las estaciones de radio. Los periodistas también fueron un blanco de las milicias y tuvieron que esconderse en el extranjero o en las regiones montañosas (Inbaraj, 1999, 22 de noviembre). Para este momento, con UNTAET ahora administrando Timor Oriental, eran notables los efectos negativos del vacío de información: había

5. Según Durand (2009: 113), el primer periódico editado en Timor-Leste surgió en 1948 y se trató del bimensual Seare, editado por la diócesis de Dili. 
rumores, pánico, desinformación, desconfianza, sensación de abandono y la presencia de grupos paramilitares.

La década de 2000 marcó la llegada de las OGI (me refiero a las sucesivas misiones de Naciones Unidas y a sus agencias) y otros actores internacionales para proporcionar ayuda humanitaria y para el desarrollo en Timor Oriental. El principal objetivo ha sido el mantenimiento de la paz (peacekeeping) y la asistencia en la construcción de las instituciones gubernamentales. El fortalecimiento de la sociedad civil y los medios locales fue contemplado como parte de la estrategia. Las actividades para el desarrollo de los medios se incluyeron en la meta denominada "incrementar la cohesión social y la participación ciudadana". Esta meta formaba parte del área de gobernanza democrática de la misión de la ONU, cuyo objetivo es "hacer la gobernanza democrática más eficiente, transparente, rendidora de cuentas y sensible para la gente" (UNDP, 2009b: 4).

El servicio radiofónico de Naciones Unidas continuó con el nombre de Radio UNTAET. Mientras Radio UNTAET estuvo en funcionamiento, una ONGI implementó el proyecto llamado Studio Moris Hamutuk ("vivir juntos"), que consistió en un programa de radio que presentaba información sobre los refugiados timorenses en la parte occidental de la isla (el Timor Occidental) y que trató de ser un canal de comunicación entre las familias que fueron separadas por el conflicto (Bratic, 2005: 117). Otro proyecto de comunicación orientado hacia la construcción de paz y la reconciliación fue la serie de televisión Istória ba futuru, de la ONG jesuita Casa de Produção Audiovisual (véase Sachse, 2009). El objetivo de este serial fue presentar hechos del pasado y leyendas tradicionales para, de esta manera, "contribuir a la búsqueda de una percepción común sobre el pasado del país” (Sachse, 2009: 56).

Radio UNTAET, por su parte, tuvo éxito en llegar a la población, aunque faltó una visión de largo plazo para "convertir este transmisora nacional de facto en una institución local viable. [...] Radio UNTAET fue concebida y administrada como un vehículo para los fines de la misión UnTAET" (Orme, 2010: 40). Después de la restauración de la independencia en 2002, los bienes de la estación fueron entregados al nuevo servicio Radio Televisão Timor-Leste, el cual "se encontró sin editores, jefes, administradores e ingenieros experimentados y sin fondos para operar" (Orme, 2010: 41).

Para 2007, las tres principales áreas de interés de desarrollo para los medios identificadas por el Programa de Naciones Unidas para el Desarrollo en Timor Oriental (UNDP, 2007) fueron la regulación, la sustentabilidad de las 
estaciones de radio comunitarias - como hemos visto, la radio es el medio al que la población tiene más posibilidades de acceder en estas condiciones - y mejorar la capacidad profesional de los periodistas. También se implementaron capacitaciones sobre cobertura electoral y de derechos humanos para los periodistas así como actividades dirigidas a mejorar el flujo de información antes, durante y después de las elecciones de 2007, entre ellas, entrega de materiales, baterías, cargadores de baterías, becas para las radios comunitarias, etcétera (NZMOM, 2007: 4).

La sociedad civil local e internacional, patrocinadas por fondos de las agencias de cooperación internacionales y con la participación de la comunidad timorense, el gobierno y los OGI, han implementado proyectos de desarrollo para los medios enfocados tanto en el entorno legal como en el desarrollo de los medios propiamente dicho.

\section{Entorno legal}

El entorno de los medios -es decir, el entorno legal e institucional - se ha desarrollado al ritmo del resto del sistema legislativo timorense, cuyo Parlamento Nacional comenzó a promulgar leyes en 2002. Como ejemplo, en 2010 aún no había un código civil, pero el Parlamento Nacional había emitido en ese año la Ley de Seguridad Nacional, de Defensa Nacional y de Seguridad Interior, entre otras. De acuerdo con IREX (2009), en algunos puntos en los que no hay ley aprobada, la legislación indonesia aún aplica. Este el caso del delito de difamación, pues ya hay casos de acusaciones de este tipo contra periodistas de investigación (Timor-Leste Law Journal, 2009). La libertad de expresión está garantizada en el artículo 40 de la constitución de Timor Oriental. El artículo 41, sobre la libertad de prensa y de medios, incluye figuras importantes, como la del secreto profesional, la libertad editorial, prohibición de los monopolios mediáticos, la libertad e independencia de los medios públicos y la existencia de un servicio público de radio y televisión. Por su parte, la última propuesta de ley de medios, depositada en el parlamento poco antes de $2010,{ }^{6}$ fue criticada por imponer restricciones al ejercicio del periodismo (edad mínima, nivel educativo, etcétera) y excepciones, niveles de clasificación y estipulaciones sobre uso de la información en el rubro del acceso a la información pública.

6. Conversación con Francisco Pinto (Sentru Internasional ba Jornalista), Dili, octubre de 2010. 
La ayuda para el desarrollo del entorno de los medios incluyó el reclutamiento de abogados y especialistas en periodismo, el análisis jurídico y la organización de foros de discusión sobre legislación de los medios y códigos de ética.

\section{Desarrollo del sistema de medios timorense}

El enfoque en el desarrollo de los medios tuvo como estrategias la construcción de capacidades y la donación de equipo e instalaciones. Las diferentes propuestas combinan estrategias difusionistas (manuales de periodismo, entrenamiento, consejería legal) con estrategias participativas (la ayuda para la instalación de estaciones de radio comunitarias, el acceso comunitario a Internet, incubación de negocios, asesoría en el desarrollo del currículum de estudiantes de periodismo y la capacitación en cascada). Estas estrategias se han aplicado en los diferentes sectores de medios — radio y televisión, medios impresos e Internet - para mejorar el acceso y las capacidades.

En cuanto a los sectores de medios, la radio es sin duda el sector más importante en el país: las principales fuentes de información de los timorenses (incluyendo comunicación impresa, electrónica e interpersonal) son Radio Timor-Leste (44.6 \%) y de boca en boca (36.3 \%) (Soares y Mytton, 2007: 13). El servicio público de transmisión auspiciado por el gobierno, RTTL, opera la estación de radio estatal (transmitida desde el distrito capital y con varias repetidoras) y un canal de televisión. Como resultado de la ayuda internacional, principalmente de la cooperación portuguesa (en la forma de entrenamiento, equipo, inversión en tecnología, contenidos de televisión, imagen gráfica), este es el servicio de información más profesionalizado del país. Se ha previsto su autonomía e independencia editorial y su vocación cultural (Jornal da República, 2010). Sin embargo, se teme sobre su falta de independencia del gobierno. La misión neozelandesa de observación mencionó:

Los transmisores públicos estatales RTL y TVTL están proporcionando un liderazgo creciente de los medios y son integrales para el crecimiento del profesionalismo del periodismo. Nos impresionó la calidad y la entrega de los canales públicos estatales. Pero los periodistas que hablaron con la misión dijeron que el estado de los canales estatales es poco claro. Algunos políticos han mostrado un compromiso insuficiente con la libertad de los medios y la responsabilidad en el periodismo que puso en duda la independencia de RTTL, el órgano que los gobierna (NZMOM, 2007: 53). 
Respecto a las audiencias de radio, la encuesta de Soares y Mytton (2007: 35) encontró que los programas de Radio Timor-Leste más escuchados por los timorenses eran los de noticias (casi 90\% de las audiencias radiofónicas las escuchaban), música (más de 40\%) y en tercer lugar hale’u distritu ("alrededor de los distritos", un programa tipo revista, más de 10\%). La mayoría de las audiencias escuchaban la radio en tetun (98\%), aunque una buena proporción también escuchaba la programación en bahasa indonesia (63.2\%). En una pregunta abierta del mismo instrumento respecto a sugerencias sobre la programación, las respuestas espontáneas más comunes fueron mejorar la transmisión de radio y/o televisión (39.2\%) y mejorar las noticias (21.4\%).

Además de RTTL, actualmente operan 15 estaciones de radio comunitarias. Ocho de ellas fueron instaladas con ayuda del Banco Mundial (NZMOM, 2007: 4). La más reciente de éstas, la Radio Maubisse-Mauloko, fue inaugurada contando con la presencia de toda la comunidad, que celebró con una ceremonia con danzantes típicos, comida y música popular. Las transmisiones de las estaciones de radio comunitarias suelen ser breves (de tres a cinco horas diarias) porque los cortes de electricidad son frecuentes en donde se ofrece este servicio. Esto obliga a las estaciones de radio a tener generadores de electricidad o paneles solares. Además, la mayor parte del personal de estas estaciones de radio trabaja voluntariamente: sin entrenamiento y sin paga, así que es difícil para ellos transmitir noticias (Article 19 and Internews 2005: 44). ${ }^{7}$

Aun con todo lo anterior, lo que el líder de la Asociación de Radios Comunitarias timorense señaló en una conversación como problema principal fue la falta de sustentabilidad, refiriendo que es sencillo obtener recursos de parte de organismos internacionales pero, paradójicamente, es al mismo tiempo difícil lograr que las comunidades participen en el mantenimiento de las estaciones. ${ }^{8}$

Los principales obstáculos para el acceso a los medios electrónicos son la electricidad —de acuerdo con Soares y Mytton (2007: 21), 34.3 \% de los usuarios de radio tienen que comprar baterías porque no gozan de servicio

7. En la capital del país hay repetidoras de varias señales extranjeras, como el BBC World Service, Radio Australia y Radio Portugal, aunque no representan fuentes significativas de información para los timorenses, pues estas estaciones tienen un nivel de audiencia inferior a $4 \%$ (Soares y Mytton, 2007: 32).

8. Conversación con Prezado Ximenes (Asosiasaun Radio Komunidade Timór Lorosa'e), Dili, octubre de 2010 . 
continuo de electricidad-; las frecuencias de radio y televisión son algunas veces inalcanzables; los receptores de radio y televisión son incosteables; y, además, los medios comunitarios e independientes tienen dificultades para sostenerse económicamente.

En cuanto a los medios impresos, los tres periódicos diarios de Timor Oriental tienen su sede en la capital y un número escaso de ejemplares se distribuye en los distritos (NZMOM, 2007). De entre los semanarios, Tempo Semanal ha trascendido por ser el primero en especializarse en el periodismo investigativo.

El costo de los ejemplares de periódicos suele ser de cincuenta centavos (el PNUD situaba a 37.19\% de la población viviendo con menos de 1.25 dólares con paridad de poder de compra). Además es muy caro producir y distribuir medios impresos en Timor Oriental: a inicios de 2010, uno de los periódicos imprimía en Indonesia y otras dos publicaciones tenían que pagar precios altos de impresión. Los salarios de los periodistas y los empleados de las empresas de medios se calculan entre 80 y 120 dólares mensuales (NZMOM, 2007: 3). Los periódicos han ido aumentando sus ventas de publicidad lentamente y con ayuda de entrenamiento de negocios para los departamentos de ventas de los periódicos (NZMOM [2007: 26] ya había observado una proporción muy baja de contenido publicitario en los periódicos). Para aliviar el problema anterior, algunos actores sociales timorenses y extranjeros consiguieron un subsidio de USAID y UNESCO para fundar una imprenta independiente que contribuya a abaratar la impresión en el país y, por lo tanto, disminuir los precios al consumidor, mejorar salarios de los empleados así como la distribución.

Los servicios de Internet y telefonía son proporcionados por Timor Telecom, una compañía timorense-portuguesa (con una participación de $40 \%$ de Portugal Telecom) favorecida con un contrato de concesión para ser el único proveedor de telecomunicaciones en el país por 15 años. De acuerdo con la compañía, el servicio de telefonía móvil cuenta con 300 mil usuarios en el país (TT, 2010). El acceso a Internet, por su parte, muchas veces se limita al distrito capital, en donde abundan los cibercafés, y el servicio es caro (una cuenta de Internet de 128 kbits por segundo cuesta 50 dólares mensuales), lo que ha hecho que varias organizaciones aún accedan a Internet a través del VSAT (un dispositivo de conexión satelital). Una ONGI estadounidense actualmente implementa un proyecto que consiste en el mantenimiento de instalaciones que ofrecen acceso a Internet y aparatos electrónicos a los periodistas en diferentes localidades del país con el objetivo de mejorar el flujo de 
noticias entre los distritos y la capital a través del envío de notas periodísticas, imágenes y audio. Otro proyecto implementado es un servicio de noticias en Internet que funciona como un taller para entrenar a periodistas en el uso de medios audiovisuales y alternativos.

Una de las condiciones indispensables para que los medios tengan efectos positivos en el desarrollo y el buen gobierno es el acceso generalizado. Una fuente de información importante en los distritos, en donde circulan muy pocos ejemplares de periódicos, son las estaciones de radio comunitarias y las repetidoras de la radio estatal. Los rumores han sido, sin embargo, importantes porque no hay muchas fuentes de información: Soares y Mytton (2007: 43) encontraron que el "de boca en boca" fue la fuente más importante de información durante la ya mencionada crisis de 2006. Pero los rumores pueden propagar pánico. Engel describió la problemática anterior de la siguiente manera (2008: 42):

Exacerbados por la poca información impresa, los bajos niveles de alfabetización y la deficiente y costosa infraestructura de las comunicaciones, los rumores sirven de base para divulgar información en el país. Ello reduce, aún más, la capacidad individual para adoptar decisiones con cierto fundamento. El pánico cunde con rapidez gracias a los rumores, sobre todo porque hay una población inquieta y preocupada por la supervivencia. No existe un organismo oficial responsabilizado con divulgar las noticias. Como el proceso de descentralización avanza a paso de tortuga, toda la información debe solicitarse al nivel nacional. Esto aumenta la sensación de carecer de derechos civiles y contribuye a la falta de unidad y objetivo común.

Durante los disturbios ocurridos en 2002, corrieron rumores de que el presidente había sido baleado. Varias veces en 2006 y 2007 hubo rumores en los distritos de que Dili "había quedado reducida a cenizas", y que "la guerra" se estaba trasladando de la capital a los distritos. Ninguna de esas informaciones resultó cierta y sólo gracias a los comentarios orales se disiparon los temores y se aclaró la información errónea.

Una misiva de la ONG local La'o Hamutuk (2006) describe los elementos que influyeron en la diseminación del pánico en referencia a la crisis de 2006 y a las personas que abandonaron en ese periodo la capital (Dili) por temor:

La mayor parte del miedo de la gente se basa en sus experiencias pasadas —no sólo 1999 sino 24 años de atrocidades militares indonesias- más que en verdadera evidencia o la realidad actual. Es verdad que este incipiente go- 
bierno debería haber manejado las cosas de mejor manera, y que tantos años de entrenamiento por asesores extranjeros han fallado en impartir principios básicos sobre control del rumor, políticas comunitarias, relación entre sector militar y comunidades, exhibición inapropiada de armas largas, priorizar las preocupaciones del público y usar los medios para mantener la calma. Nada se ha hecho para enseñar a la gente común acerca de estrés post traumático. Pero deberíamos darnos cuenta de que el pánico no implica que tras el miedo haya un motivo racional, especialmente entre personas traumatizadas con escasas reservas psicológicas o materiales.

Muchos comentaristas de la actual situación refieren el 4 de diciembre de 2002 o el año 1999, dos periodos recientes en los que grupos de hombres violentos diseminaron el pánico en Dili. El 2 de enero de 2005 es igualmente relevante. En ese día, una semana después del tsunami en Aceh, ${ }^{9}$ un pequeño grupo de personas diseminaron rumores en Dili y zonas costeras cercanas acerca de que un tsunami estaba a punto de impactar y matar a todos. Después de que muchedumbres se refugiaron en las montañas (sin creer las garantías de la policía de que no había tsunami), muchas casas fueron saqueadas.

Además de acceso, las audiencias requieren que los medios sean de calidad. Como se mencionó, una de las justificaciones del énfasis en el desarrollo de los medios es el temor de que los medios propaguen mensajes de odio o magnifiquen el pánico. Por el contrario, practicantes prevén diversas funciones (positivas) de los medios durante crisis o conflictos. Al respecto, algunos actores han referido que en Timor los periodistas suelen temer a los servidores públicos (son excesivamente respetuosos), frecuentemente construyen sus reportes en torno a declaraciones y figuras de autoridad y además son inexactos o con mala redacción: hay "inexactitudes en los periódicos aún cuando la información venía de un comunicado de prensa" (NZMOM, 2007: 9). El reporte de la misión de observación de medios neozelandesa formada con ocasión de las elecciones de 2007 atribuía esto a que los periodistas "eran jóvenes y necesitaban mucho más entrenamiento" y que "confrontar la autoridad o cuestionar las fuentes [...] los hacía sentir temerosos e incómodos" (NZMOM, 2007: 9).

El poder de los medios impresos en Timor Oriental, a pesar del difícil acceso de la población, ha quedado constatado por los escándalos políticos

9. Un territorio especial indonesio ubicado en la isla de Sumatra, al oeste del archipiélago. Este escenario volvió a repetirse tras el tsunami de Japón en marzo de 2011 (Tempo Semanal, 2011, marzo). 
ocasionados por la filtración de comunicaciones privadas de miembros del gabinete de Estado. Otro ejemplo fue la publicación de una nota que incluyó, presumiblemente, algunos comentarios off the record de un jefe de policía sobre una actividad ilegal realizada por un veterano de guerra. La respuesta del veterano fue llanamente sugerir que el periódico que la publicó debía ser cerrado por el gobierno. Otros casos que trascendieron en este periodo fueron la exigencia de una red de ciudadanas para que los medios reporten éticamente los delitos sexuales - de una manera que no dañe psicológicamente a las víctimas y sus familiares (Diario Nacional, 29 de julio de 2010, véase Timor-Leste Media Development Center, 2010) - y la exigencia de la ONG local Luta Hamutuk ("luchar juntos") para tener mayor equidad de género en los reportes de los medios así como también permitir que la gente ordinaria exprese también sus opiniones (Radio Timor-Leste, 19 de agosto de 2010, véase Timor-Leste Media Development Center, 2010).

\section{Conclusiones}

Entre las justificaciones de la categoría de "desarrollo para los medios" como parte de la ayuda para el desarrollo en sociedades con conflicto podemos identificar, por un lado, los efectos positivos de los medios en el desarrollo humano y el buen gobierno - aunque, como mencioné, en el estudio de Norris y Zinnbauer (2002) estos efectos parten del supuesto que la gente en general tiene acceso a los medios y que los canales de comunicación son independientes-, pero, además, argumentar que los conflictos pueden ser empeorados cuando se disemina información engañosa o que incita a la violencia. Al contrario de los efectos negativos de los medios en los conflictos, ampliamente ejemplificados con lo sucedido en varios conflictos armados, los efectos positivos son materia de debate entre comunicólogos y practicantes. Mientras algunos dudan de este tipo de potencial (Hamelink, 2008), ${ }^{10}$ otros centran sus esfuerzos en definir los requerimientos de intervenciones comunicacionales (e. g. proyectos de medios) durante fases concretas del conflicto (v. gr. Becker, 2004).

10. Hamelink (2008) se centra en la necesidad de desarrollar un "sistema de alerta de los medios internacionales" que monitoree los medios en zonas de conflicto para identificar señales tempranas de incitación al genocidio. 
Muchos esfuerzos se han dirigido a ayudar a que los medios locales timorenses adquieran la capacidad de proveer acceso a la información en todos los distritos del país de una manera independiente, democrática, responsable y sostenible. Estos esfuerzos han sido realizados por actores tanto timorenses como extranjeros a partir de cero, pues la infraestructura de comunicaciones fue destruida (y los periodistas locales perseguidos) en los eventos violentos de 1999, año de la consulta popular. Los logros — por ejemplo, la profesionalización y capacidad de cobertura de RTTL, la puesta en marcha de radios comunitarias - han sido loables, especialmente si consideramos que se ha incentivado la pluralidad de la información en un mercado muy pequeño, reducido aún más por la pobreza y el analfabetismo.

Con todo, diferentes problemas de información persisten al lado de los demás referentes al desarrollo: centralización en el distrito capital (al igual que los servicios públicos), obstáculos para lograr ser negocios sustentables (como en otros sectores productivos) y criterios de calidad y de condiciones de trabajo aún por alcanzar. my

\section{Referencias}

Article 19 e Internews, Freedom of the expression and the media in Timor-Leste. Londres, 2005, 119 pp., en: www.article19.org/pdfs/publications/timorleste-baseline-study.pdf. (recuperado el 7 de octubre de 2010).

Becker, Jörg (2004), "Contributions by the media to crisis prevention", Conflict and Communication Online, núm. 1-2, Verlag Irena Regener, Berlín.

Bratic, V., In search of peace media: examining the role of media in peace developments of the post-cold war conflicts. Tesis doctoral. Ohio, Ohio University, 2005.

Bratic, V. y Schirch, Why and when to use the media for conflict prevention and peacebuilding? Holanda, European Centre for Conflict Prevention-Global Partnership for the Prevention of Armed Conflict, 2007.

Durand, F., História de Timor-Leste. Da pré história à actualidade, Lisboa, Lidel, 2009.

East Timor Law \& Justice, “JSMP: Use of Portuguese language makes it harder for a number of parliamentarians to debate the civil draft code". 31 de julio de 2010, en: http://easttimorlegal.blogspot.com/2010/07/jsmpuse-ofportuguese-language-makes-it.html (recuperado el 7 de octubre de 2010). 
Engel, R. E., "Timor-Leste: contribuciones internacionales a un Estado débil”, Temas, núm. 53, pp. 33-44, La Habana, marzo de 2008.

Gillete, R., "Media matters: professionalizing and regulating media in postconflict Bosnia and Kosovo", Woodrow Wilson International Center for Scholars. Meeting report 324, 2006, en: http://www.wilsoncenter.org (recuperado el 23 de septiembre de 2010).

Hamelink, C., "Media between warmongers and peacemakers", Media, War and Conflict. 1. Estados Unidos, Sage y Annenberg School for Communication, 2008.

Hicks, D., "Ema lorosa'e, ema loromonu: Identity and politics in Timor-Leste", Christine Cabasset-Semedo y Frédéric Durand (eds.), East Timor. How to build a new nation in Southeast Asia in the 21st century?, Institute de Recherche sur l'Asie de Sud-Est Contemporaine, 2009.

Howard, R., Conflict sensitive reporting: State of the art, París, Unesco, 2009. Hyer, R. N., y V. T. Covello, Effective media communication during public health emergencies, Génova, World Health Organization, 2005.

Inbaraj, S., "East Timor journalists start to rebuild media". Mensaje a la lista de correo ETAN. 22 de noviembre de 1999, en: https://lists.riseup.net/ www/arc/east-timor (recuperado el 23 de septiembre de 2010).

IREX, Media sustainability index 2008, 2009, en: http://www.irex.org (recuperado el 7 de octubre de 2010).

Jornal da República, "Política Nacional para a Comunicaçao Social”, serie 1, núm. 13. República Democrática de Timor-Leste. 31 de marzo de 2010.

La'o Hamutuk. "Panic and reality in Dili", 6 de mayo de 2006, en: http:// www.laohamutuk.org/Justice/Panic/06LHFDTL.html (recuperado el 6 de noviembre de 2010)

La'o Hamutuk. "Timor-Leste must win independence from petroleum / Remarks at launch of the UNDP 2011 Timor-Leste Human Development Report by Charles Scheiner, La'o Hamutuk", 3 de mayo de 2011, en: http://www.laohamutuk.org/econ/HDI10/LHatUNDPNHDRlaunch3May2011En.pdf (recuperado el 7 de mayo de 2011).

New Zealand Media Observation Mission, Report on 2007 Timor-Leste Elections, 2007, en: http://www.pmc.aut.ac.nz/ docs/papers/ NZtimorreport_2007Final.pdf (recuperado el 23 de septiembre de 2010). Norris, P., y D. Zinnbauer, Giving voice to the voiceless: Good governance, human development and mass communications, United Nations Development 
Programme, 2002, en: hdr.undp.org/en/reports/global/hdr2002/papers/ norris-zinnbauer_2002.pdf (recuperado el 6 de noviembre de 2010).

Orme, B., Broadcasting in UN Blue: The unexamined past and uncertain future of peacekeeping radio, Estados Unidos, Center for International Media Assistance y National Endowment for Democracy, 2010.

Price, M. E., "Restructuring the media in post-conflict societies: Four perspectives. The experience of intergovernmental and non-governmental organizations", Cardozo Online Journal of Conflict Resolution, 2000.

Price, M. E. y P. Krug, The enabling environment for free and independent media / Contributions to Transparent and Accountable Governance, Estados Unidos, Office of Democracy and Governance/USAID, 2002.

Sachse, H., "Reconciliation in Timor-Leste and the role of the media: The Casa de Produçao Audiovisual", Christine Cabasset-Semedo y Frédéric Durand (eds.), East Timor. How to build a new nation in Southeast Asia in the $21^{\text {st }}$ century?, Institute de Recherche sur l'Asie de Sud-Est Contemporaine, 2009. Soares, E. y Mytton, G. Timor-Leste National Media Survey Final Report. Foundation Hirondelle, 2007, http://timor-leste.usaid.gov/publications/ USAID\%20Timor-Leste\%20National\% 20Media\%20Survey\%20Final\%20 Report\%202007.pdf (recuperado el 14 de mayo de 2010).

Taylor-Leech, K., "Language and identity in East Timor: The discourses of nation building", Language Problems and Language Planning, vol. 32, núm. 2, Amsterdam, John Benjamins Publishing Company, 2008.

Tempo Semanal, "Tsunami in Japan. Horta get angry at Timorese Secretary State for Security and send his support to Japanese people", marzo de 2011, en: http://temposemanaltimor.blogspot.com/2011/03/tsunamiin-japan-horta-get-engry-at.html (recuperado el 10 de mayo de 2011).

Timor-Leste Law Journal, “Defamation, a crime or not in Timor-Leste?”, TimorLeste Law Journal, abril de 2009, en: http://www.eastimorlawjournal.org/ ARTICLES/ 2009/Defamation_in_Timor_Leste_A_Crime_or_Not.html (recuperado el 7 de octubre de 2010).

Timor-Leste Media Development Center, “Timor News Line”, 2010, en: http:// timornewsline.com (recuperado el 6 de noviembre de 2010).

Timor Telecom, "Chronology", 2010, en: http://www.timortelecom.tp (recuperado el 23 de septiembre de 2010).

USAID, The role of media in democracy: a strategic approach, junio, Washington, USAID, 1999, en: http://www.usaid.gov (recuperado el 7 de octubre de 2010). 
UNDP, Human Development Report 2009: Indicators, 2009a , en: http://hdrstats. undp.org/es/indicators (recuperado el 23 de septiembre de 2010).

UNDP, UNDP Country Programme for Timor-Leste 2009-2013, 2009b, en: http:// www.tl.undp.org (recuperado el 7 de octubre de 2010).

UNDP, Human Development Report 2010: Indicators, 2010, en: http://hdrstats. undp.org/en/data/map (recuperado el 20 de enero de 2011).

UNDP Timor-Leste. Timor-Leste independent media development initiative, diciembre de 2007, en: http://www.tl.undp.org/UNDP/what\%20we\%20 do/Democratic\%20Governance /Media/Signed\%20Pro\%20Doc.pdf (recuperado el 7 de octubre de 2010).

Waisbord, S., "The irony of communication for social change", Mazi, 12, agosto de 2007, en: http://www.communicationforsocialchange.org/ mazi-articles.php?id=349 (recuperado el 23 de septiembre de 2010). 